\title{
Metabolomics Used in Herb-Induced Liver Injury
}

\author{
Geng Xingchao*2, Wu Wenxiao ${ }^{1,2}$, Wang Ting ${ }^{2}$ and Li Bo ${ }^{2}$ \\ ${ }^{1}$ Chinese Academy of Medical Sciences \& Peking Union Medical College, China \\ ${ }^{2}$ National Institutes for Food and Drug Control, Beijing, China
}

Received: March 20, 2018; Published: April 05, 2018

*Corresponding author: Geng Xingchao, National Center for Safety Evaluation of Drugs, National Institutes for Food and Drug Control, Key Laboratory of Beijing for Nonclinical Safety Evaluation of Drugs, Beijing 100176, China, Email: gengxch@nifdc.org.cn

Abbreviations: MS: Mass Spectrometry; TCM: Traditional Chinese Medicine; NMR: Nuclear Magnetic Resonance; HILI: Herb-Induced Liver Injury; AA: Aristolochic Acid

\section{Introduction}

Metabolomics [1]. As a formidable tool, is usually used to systematically assess the molecular responses of living systems to all external stimuli, characterize pathological states, diagnostic information and mechanistic insights into biochemical effects of drugs, based on global metabolite profiles in biological samples [2]. Mass spectrometry (MS) and nuclear magnetic resonance (NMR) spectroscopy, two major analytical platforms, with visualization software, have driven forward the discipline of metabolomics. Traditional Chinese Medicine (TCM) with its focus on herbal use is popular and appreciated worldwide with increased tendency $[3,4]$. And treatment was perceived as fairly safe but discussions emerged more recently as to whether herb-induced liver injury (HILI) from herbal TCM is a major issue. The multi-component and multi-target nature of TCM makes toxicological research far more complex than that of chemical drugs. Looking for rapid and sensitive biological toxicity effect evaluation technology has become a major problem urgently to be solved in current TCM safety evaluation. Metabolomics can further be employed as a perfect approach to link the gap between TCM and molecular toxicology. We reviewed the applications of the metabolomics with two major analytical platforms for HILI researches.

\section{NMR-Metabolomics Used in HILI}

NMR is one of the most commonly used technologies in metabolomics research, providing detailed information on the molecular structure and probing metabolite molecular dynamics and mobility [5]. Aconite is a very typical toxic Chinese medicine. Li et al. [6]. Using [1] H NMR for investigation of metabolites of Aconite on its toxicity in Westar rats. The 1H NMR spectral analyses were coincident with the results of serum biochemical parameters which mean the slight impairment of liver. The sub-acute toxicity of aristolochic acid (AA) was investigated by $1 \mathrm{H}$ NMR spectroscopic by Pei Lab [7]. Slight liver lesion was observed in spectra of AA, a main component of Aristolochia and As arum, treated rats in time interval of 48-56 $\mathrm{h}$ and 96-104 $\mathrm{h}$ based on the increase of urinary turbine and decrease of 2-oxoglutarate, which was NMR marker of liver impairment.

\section{MS Metabolomics Used in HILI}

The high selectivity of mass spectrometry with low-detection limits makes mass spectrometry an ideal tool for metabolomics applications. It is a sensitive tool that can be used to characterize, identify and quantify a large number of compounds in a biological sample where metabolites concentrations might cover a broad range of information with regard to disease path physiology [8,9], Zhao and her coworker [10]. Using LC-MS for investigation of metabolites of Triptergium wilfordii on liver toxicity of Sprats analyzed and identified seven possible endogenous biomarkers, which were 6-phosphate glucosamine, lysophospholipid, tryptophan, guanidine acetic acid, 3-indole prop ionic acid, cortisone, and ubiquinone. The level changes of above metabolites indicated the related metabolism pathways were disordered. The toxic effect of total spooning had been established by using UPLCMS. It was shown that there was obvious acute liver toxicity and accumulated one for saikosaponin Wang et al. [11,12].

Evaluated dynamic metabolomics changes of liver injury in rats caused by Polygonal multifulorum (PM) with LC-MS/MS-QTOF. Six potential biomarkers were screened through comparative analysis, including polyamide, Lysophosphatidylcholines (16:0), leukotriene A4, trans-tetra-dec-2-enoicacid, dihydrocortisol and 7a-hydroxydehydroepiandrosterone, which are more sensitive than ALT and AST. It can reveal the dynamic mechanism of PM causing liver injury. Cui et al. [13]. Discovered that 55 metabolites distributed in 33 metabolic pathways were identified as being 
significantly altered in Dioscorea bulbiferous Rhizome-treated rats using UPLC-Q-TOF/MS. Dong et al. [14]. revealed that Aconite Root produced serious heart and liver toxicity by analyzed the urinary metabolomics which was performed by UPLC-Q-TOFHamlin Westar rats, consistent with clinical biochemistry and histopathology. Furthermore, urine metabolomic profiling has provided 17 sensitive biomarkers, The rat urine after given Fructus Conidia were analyzed by UPLC/Q-TOF MS indicates that the extractor of Fructose Conidia has certain toxicity to the liver and kidneys [15].

\section{Conclusion}

The metabolomics analysis were coincident with the results of classical toxicology study parameters, even more superiority to traditional technologies on the toxicological study of TCM with the abilities of dynamical monitoring the response of bio system and evaluating biological effects from the metabolic [6]. Due to the complexity of HILI mechanism, metabolomics is especially suitable on the hepatic toxicity study of traditional Chinese medicine.

\section{References}

1. Nicholson JK, Lindon JC, Holmes E (1999) Metabonomics: understanding the metabolic responses of living systems to pathophysiological stimuli via multivariate statistical analysis of biological NMR spectroscopic data. Xenobiotica 29(11): 1181-1189.

2. Wang R, Xiong AZ, Teng ZQ Yang QW, Shi YH, et al. (2012) Radix Paeoniae Rubra and Radix Paeoniae Alba Attenuate CCl4-induced acute liver injury: an ultra-performance liquid chromatography-mass spectrometry (UPLC-MS) based metabolomic approach for the pharmacodynamic study of Traditional Chinese Medicines (TCMs). Int J Mol Sci 13(11): 14634-14647.

3. Wu WY, Yang WZ, Hou JJ, Guo DA (2015) Current Status and Future Perspective in the Globalization of Traditional Chinese Medicines. WJTCM 1(1): 1-4.

4. Leonti M, Casu L (2013) Traditional medicines and globalization: current and future perspectives in ethnopharmacology. Front Pharmacol 4: 92.

5. Moolenaar SH, Engelke UF, Wevers RA (2003) Proton nuclear magnetic resonance spectroscopy of body fluids in the field of inborn errors of metabolism. Ann Clin Biochem 40(1): 16-24.
6. LI L, Sun B, Zhang Q, Fang J, Ma K, et al. (2008) Metabonomic study on the toxicity of Hei-Shun-Pian, the processed lateral root of Aconitum carmichaelii Debx. (Ranunculaceae). J Ethnopharmacol 116(3): 561568.

7. Zhang X, Wu H, Liao P, Li X, Ni J, et al. (2006) NMR-based metabonomic study on the subacute toxicity of aristolochic acid in rats. Food Chem Toxicol 44(7): 1006-1014.

8. Tolstikov VV, Fiehn O, Tanaka N (2007) Application of liquid chromatography-mass spectrometry analysis in metabolomics: reversed-phase monolithic capillary chromatography and hydrophilic chromatography coupled to electrospray ionization-mass spectrometry. Methods Mol Biol 358: 141-155.

9. Fiehn $O$ (2008) Extending the breadth of metabolite profiling by gas chromatography coupled to mass spectrometry. Trends Analyt Chem 27(3): 261-269.

10. Zhao X, Liu X, Xu C, Ye T, Jin C, et al. (2015) Investigation of metabolites of Triptergium wilfordii on liver toxicity by LC-MS. China J Chin Mater Med 40(19): 3851-3858.

11. Lv T, Mou H, Fen J, Zhu X, Liu L, et al. (2013) Metabolomics on acute liver toxicity of saikosaponin in Chinese medicine bupleuri radix. Chem Res Appl 25: 789-792.

12. Ma Z, Li Q, Zhao K, Wang J, Xiao X (2017) Dynamic serum metabolomics study of liver injury in rats caused by Polygonum multifulorum. China J Chin Mater Med 42(1): 152-156.

13. Zhao DS, Jiang LL, Fan YX, Wang LL, Li ZQ et al. (2017) Investigation of Dioscorea bulbifera Rhizome-Induced Hepatotoxicity in Rats by a Multisample Integrated Metabolomics Approach. Chem Res Toxicol 30(10): 1865-1873.

14. Dong H, Zhang A, Sun H, Wang H, Lu X, et al. (2012) Ingenuity pathways analysis of urine metabolomics phenotypes toxicity of Chuanwu in Wistar rats by UPLC-Q-TOF-HDMS coupled with pattern recognition methods. Mol Biosyst 8(4): 1206-1221.

15. Han L, Feng Y, Jiang T, Wang L, Zhao J, et al. (2012) Study on Metabonomics and Hepatic-renal Toxicity of Extractum Fructus Cnidii Made by Supercritical Fluid Extraction. Tradit Chin Drug Res Pharmacol 23: 131-135.
(C) (i) This work is licensed under Creative Submission Link: https://biomedres.us/submit-manuscript.php

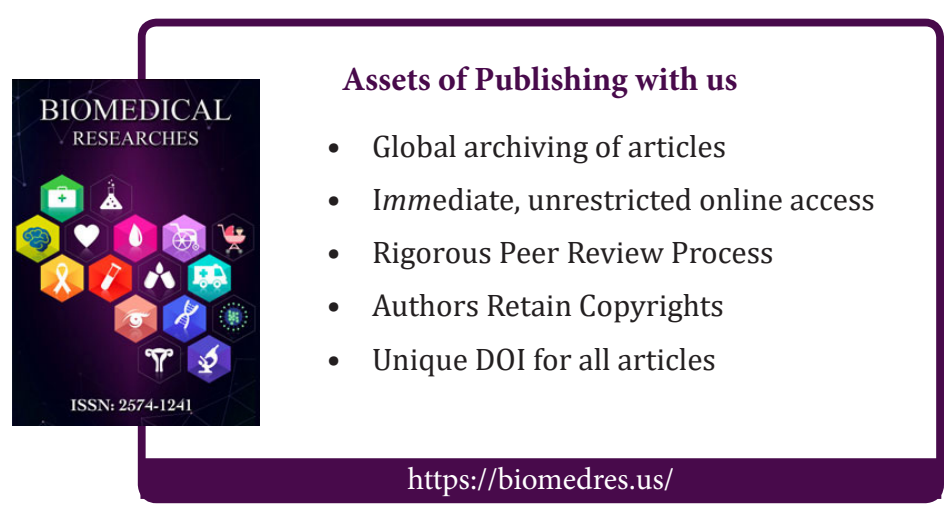

